\title{
User-Oriented Product Development with Advanced Scan Solutions
}

\author{
Huangmei LIN*, Ellen WENDT, Doudou ZHANG, Jessica BOLL, \\ Jana SIEGMUND, Sybille KRZYWINSKI, Yordan KYOSEV \\ TU Dresden, Institute of Textile Machinery and High Performance Material (ITM), Germany \\ https://doi.org/10.15221/21.12
}

\begin{abstract}
Recent developments in scanning technology have enabled researchers to scan persons at up to 180 frames per second with an accuracy of less than $1 \mathrm{~mm}$ during movement. This allows a highly precise analysis of the interaction between body and textile as well as deformations of the fabric during movements. With the Move4D scanner, dynamic movement can be recorded and analysed very quickly. Thus, the authors are capable of making a significant contribution to the improvement of functional (e.g. sports and medical) and protective clothing by a digital process chain. The result of this research can be used for the applications on the product development of the tight-fitting and loosefitting garments. Advantages of the digital linked process chain based on scanner are as follows: i) The design cycle of clothing is shortened by early simulation. The influence of the material behaviour on the product design can be analysed at an early stage; ii) Modification between the 3D development and the 2D patterns is convenient and intuitive; iii) Sophisticated digital data management helps to increase the efficiency of the entire process chain.
\end{abstract}

Keywords: Advanced scanning technology, User typical body position, Processing, 3D fit simulation

\section{Introduction}

The research activities of the professorship of Development and Assembly of Textile Products at the Institute of Textile Machinery and High Performance Material (ITM) are focused on the development of machines and technologies for the textile and clothing industry, on material modeling and on (digital) product development. One of the most important prerequisites for digital product development are CAD data.

Advanced scanning technologies simultaneously capture and digitize the shapes and textures of any object. The Move4D high-speed 4D scanner from the Instituto de Biomecanica in Spain [1] has been available at the ITM since December 2020 for dealing with innovative research projects related to human and their clothing. The high-speed scanner records the entire body with up to $180 \mathrm{~Hz}$ and an accuracy of less than $1 \mathrm{~mm}$. This enables a highly precise analysis of the interaction between the body and the textile as well as the deformation during movements. With the Move $4 D$ scanner, dynamic movement can be recorded and analysed very quickly. As a result, the team of professorship is able to make a significant contribution to increasing efficiency in product development and to improving functional clothing (e.g. sports).

In addition to accurate body data, knowledge of the behavior of the flexible material used is essential. Materials are one of the four fundamental factors (together with function, aesthetics and manufacturing processes) in design decisions for every product. Such decisions are difficult because the information about textile materials is complex [2].

In this paper, we develop a continuous digital process chain from product idea to product and quality monitoring. It is important to overcome the digital gap between design process and prototyping in order to enable early communications and interactions. This contains:

- Scanning,

- Design decision,

- Pattern development in 2D or 3D,

- Determination and consideration of material parameters,

- Visualization of the product.

The main contribution of this paper falls into two proposed orientations for the product development, as shown in Figure 1, with 3D construction solutions (in red) and using 2D CAD solutions (in green). Kinematic human models based on scanning data offer another alternative for product development (in blue).

* huangmei.lin@tu-dresden.de; +49- 351- 46339327; http://tu-dresden.de/mw/itm 


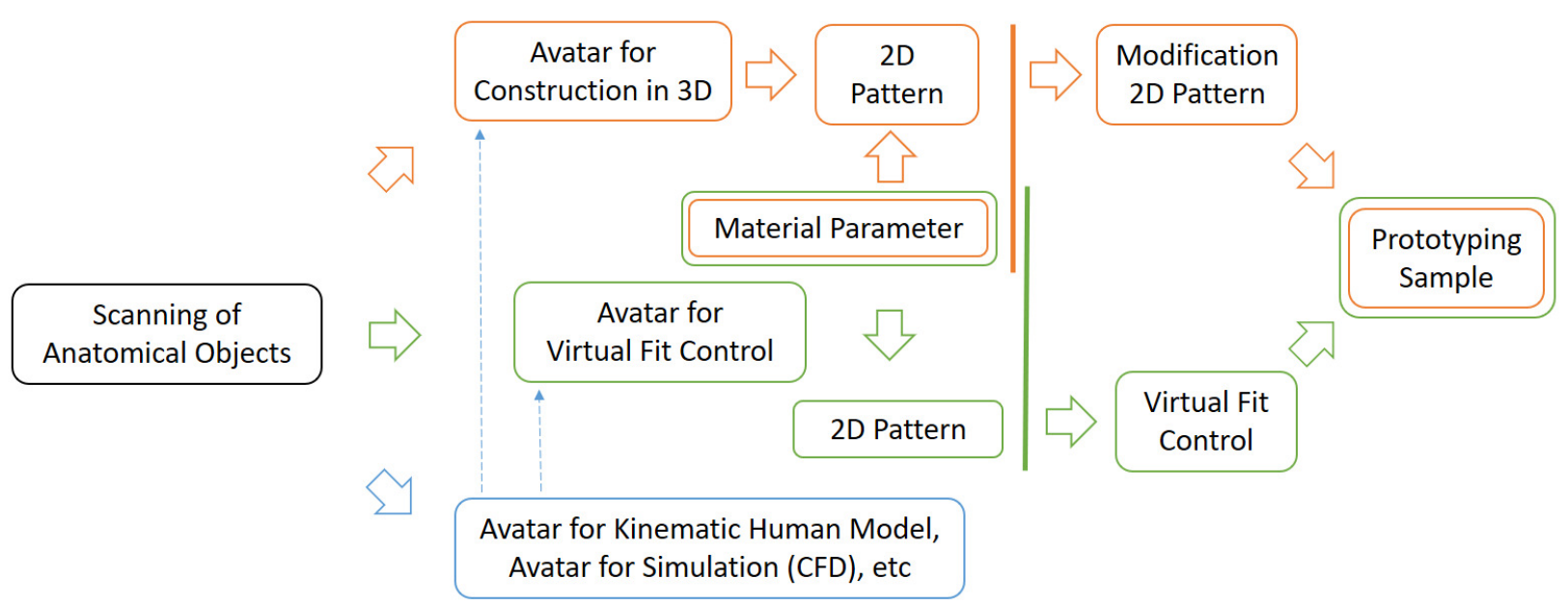

Fig. 1. Process chain of virtual product development for low stiff materials based on scanning

\section{Methods and procedures with 3D construction solutions}

The digital product development with 3D construction solutions is preferably used for tight-fitting garments (e.g. cycling race suits, body flying suits [3, 4]). This process chain (Fig. 1, marked in red) from scanning in standard pose and in motion, preparing the body data e. g. for aerodynamic simulations, the construction in $3 \mathrm{D}$ to pattern cuts in $2 \mathrm{D}$ considering the material properties was developed to meet the requirements for accurately fitting functional clothing.

Aerodynamics plays a major role in speed sports such as cycling. To be successful in highperformance sports, all components must be optimally coordinated with one another. In addition to the technical equipment (in cycling, these are the bike, helmet, shoes), this also applies to the athlete's pose and movement. The functionality of the racing suit has been recently intensively investigated and adapted to the respective requirements. This concerns both the design of the suit and the surface properties of the textile material.

For the aerodynamic functionalisation of cycling clothing, warp-knitted fabrics with defined fluidic behaviour were used. These warp-knitted fabrics were positioned on the body with the aid of simulations, taking into account the typical motion sequence in this sport. The aim is to reduce flow resistance and thus increase the cyclist's performance [3].

\subsection{Generation of scan data}

\subsubsection{Generation of body data in racing pose}

Scanning a person in cycling position is challenging due to shading during the movement of the human body and the bicycle. Therefore, we suggest two methods to achieve the necessary body data for the further work both to produce the test dummy for the wind tunnel tests and the aerodynamic simulation.

On the one hand, the test person was scanned in a standard pose with a 3D body scanner (Artec Leo, zSnapper ${ }^{\circledR}$ cart (Vialux)). A kinematic human model - SMPL model ("A Skinned Multi-Person Linear Model" - Max Planck Institute, Tübingen/Germany) was used to create the final geometry data. This model makes it possible to transfer the body shape of the test person into the desired pose by means of an internal skeleton. On the other hand, the body was scanned directly in the typical pose. This data set was used for the final correction of the data set previously animated with the SMPL model [5]. 


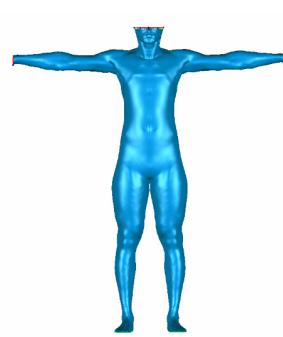

Scan data in basic pose

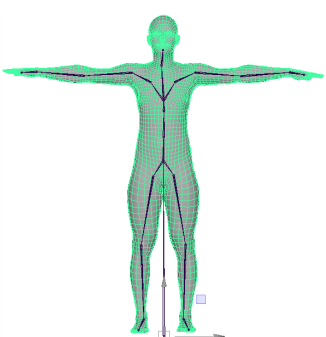

Implementation of a skeleton
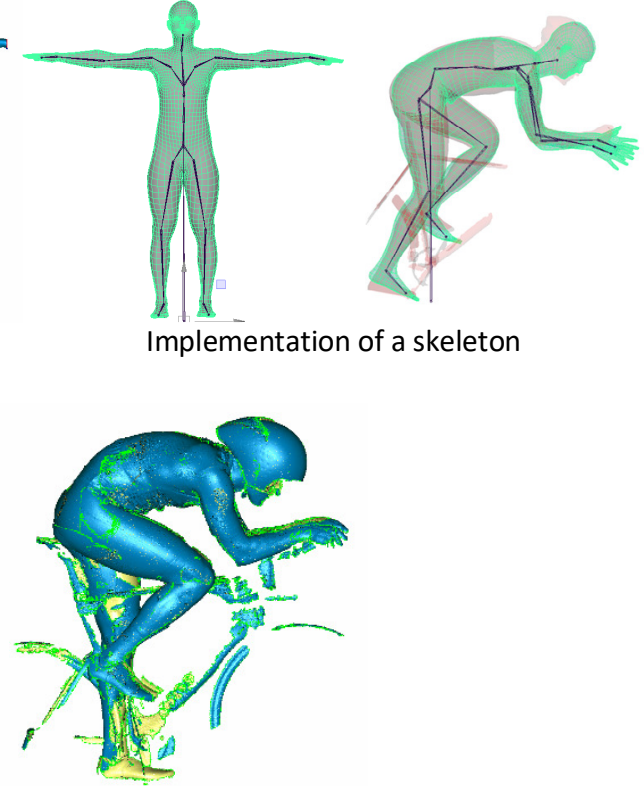

Scan data in racing pose (Leg position in $0^{\circ} / 180^{\circ}$ )

(a)

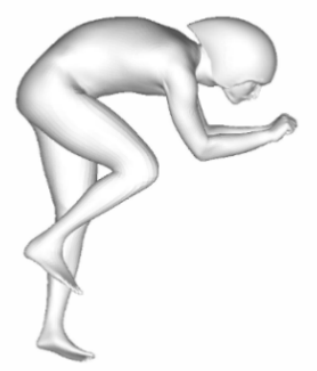

(a) Preparation of the body data by means of a kinematic human model

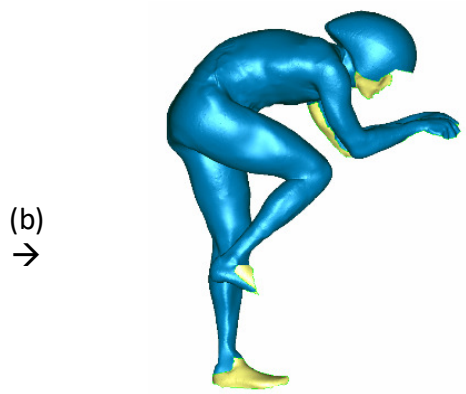

(b) Preparation of the body data from scanning

Fig. 2. Capture and preparation of the body data in racing pose for production of test dummy and aerodynamic simulation (a) by means of a kinematic human model (b) from scanning

Figure 3 shows the correction of the kinematic human model through fine tuning with the help of scan data in the pose of the cyclist with leg position in $0^{\circ} / 180^{\circ}$. Thus, both the curvature of the back and the posture of the head as well as the hands could be corrected.

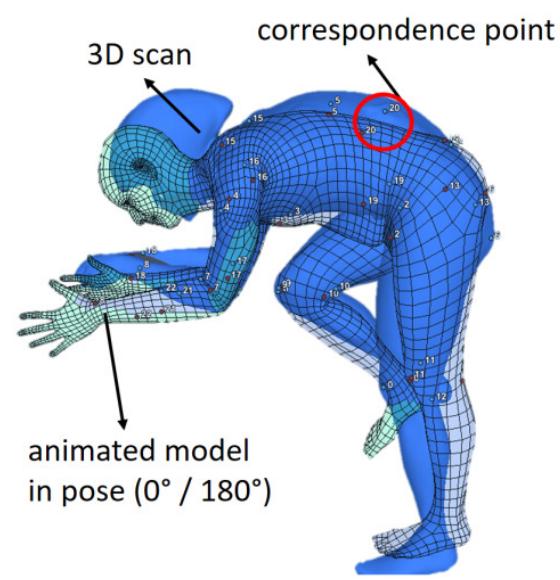

Fig. 3. Fine tuning: correction of the kinematic human model comparing with scan data

\subsubsection{Generation of body data for $3 D$ pattern construction}

When scanning in motion with Move $4 D$, there is the advantage that the pose suitable for the pattern construction in $3 \mathrm{D}$ can be determined very quickly based on the recorded data. This can reduce the time you need to rescan each time. The pose of the athlete in the high performance sport (Fig. 2) is usually not identical to the pose in which the racing suit is constructed. The pattern cuts of the cycling suit should be made in such a way that the fit is optimal in the racing position, but can also be worn outside the competition. 


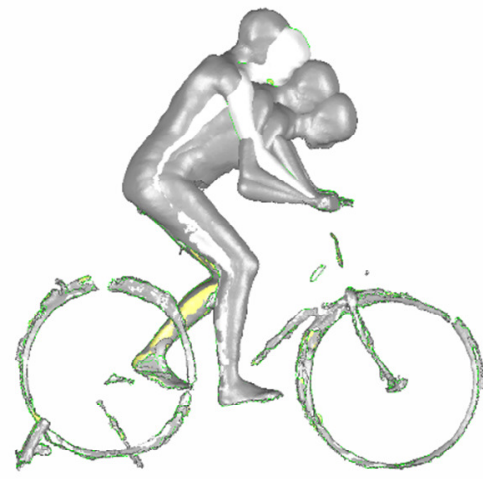

Scan data (4 frames)

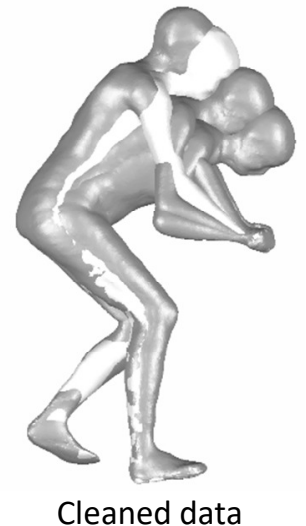

(4 frames)

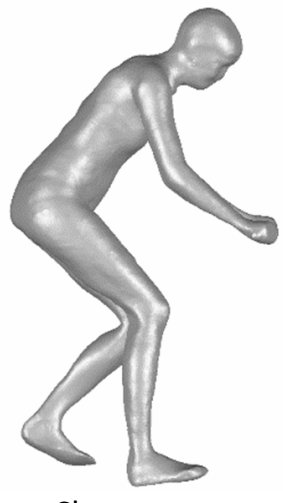

Chosen pose

Fig. 4. Capture and preparation of scan data in an upper body lift for construction of racing suit

An overview of four selected poses from the subject's scan data while raising the upper body is shown in Figure 4. For the 3D design development, the pose with $45^{\circ}$ flexion was selected.

\subsection{Construction of racing suit in $3 \mathrm{D}$, development of $2 \mathrm{D}$ pattern cuts}

The 3D body model is a prerequisite for using a $3 \mathrm{D}$ construction solution. The advantage of the construction in 3D is that the suit design can be drawn directly on the body surface (Fig. 5) and processed in a $2 \mathrm{D}$ environment. This allows reproducing the body shape exactly. The design draft in this case was made on the left half of the body, assuming that the body is symmetrical [3].

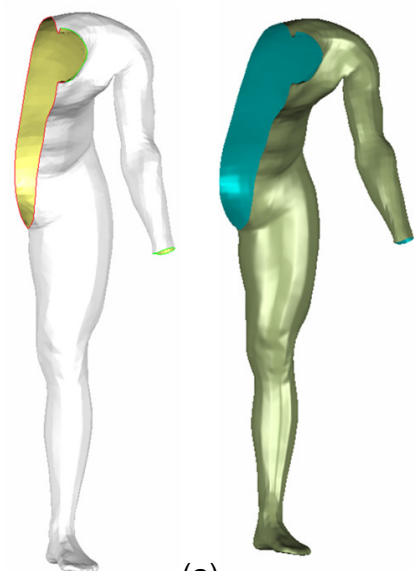

(a)

(b)
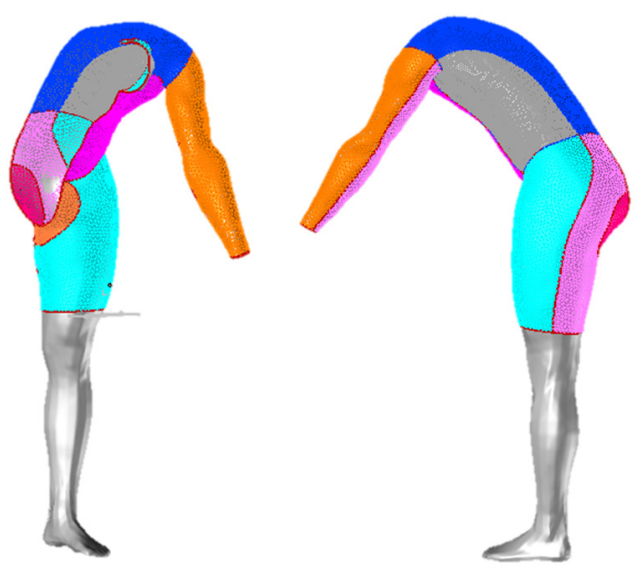

(c)

Fig. 5. Designing of racing suit in the selected pose, (a) polygon model, (b) volume model, (c) mesh within the pattern boundaries

The design draft was drawn directly on the body surface of the solid model using the software Design Concept 3D (DC3D) from Lectra. The area within these pattern boundaries was transferred into polygon meshes in $3 \mathrm{D}$ (Fig. 5, right) and then flattened into the $2 \mathrm{D}$ environment using mathematical algorithms $[6,7]$.

The pattern control was carried out by comparing the seam lengths and the areas of the pattern cuts. In addition, in DC3D the material behaviour can be taken into account. For this purpose, the stressstrain curves of the material were integrated into the software (Fig. 6, left). To achieve an optimal fit of tight-fitting sportswear, the pattern cuts are usually processed undersized. The undersize depends on the requirements for function and wearing comfort. For the racing suit, a warp-knitted fabric was used, with the pattern cuts scaled down by $15 \%$ both in the longitudinal and circumferential directions (Fig. 6 , middle). Further, the manufactured racing suit was available for further investigations in the wind tunnel, both for the test dummy and for the real cyclist (Fig. 6, right). 


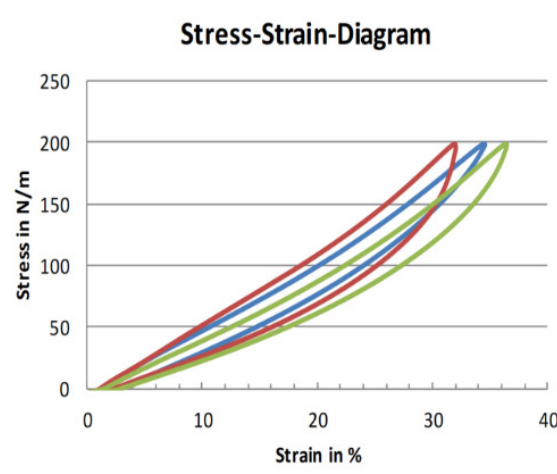

(a)

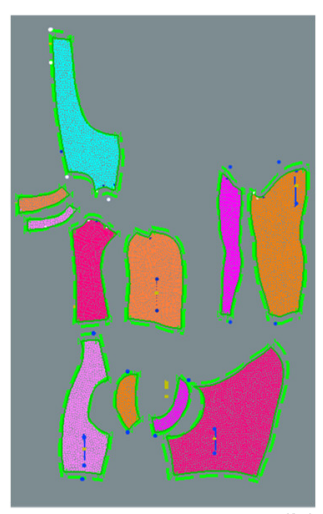

(b)

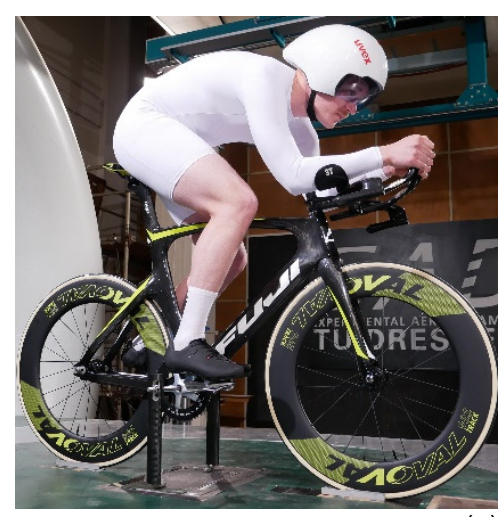

(c)

Fig. 6. Consideration of material behavior during the pattern development, (a) exemplary stress-strain curve for implementation in $D C 3 D$,(b) undersized pattern generation and (c) manufactured racing suit in use.

The pattern development based on 3D construction and the subsequent flattening in $2 \mathrm{D}$ enable both the validation of the conventionally construction in $2 \mathrm{D}$ and the exact positioning of seams and pattern cuts from different materials according to the results of the aerodynamic simulation.

This procedure accelerates the development process and hence, reduces development costs, and the time-consuming prototype manufacturing. The proposed process chain can be applied to other speed sports.

\section{Methods and procedures using 2D CAD solutions}

The procedure using 2D CAD solutions is shown in Figure 1 (in green).

For example, pattern cuts of a dress are generated with 2D CAD solutions (Modaris, Grafis, Assyst, etc.). Hereby a high degree of experience of the pattern designer is necessary in order to describe the complex body shapes with 2D pattern cuts [8].

For virtual fit control, scanning provides accurate bodies as avatars. The use of a kinematic human model allows the creation of an avatar with specific pose, if required. In addition, scanning specific motion sequences with a 4D scanner enables to select the appropriate body pose for the fit simulation.

Furthermore, the material properties influencing the fit, such as tensile behaviour, bending stiffness, and drapability, must be determined and integrated into the fit simulation software (Vidya from Assyst).

\subsection{Selection of materials and clothing}

The investigations were carried out using a Godet dress made from the material B1 listed in Figure 7.

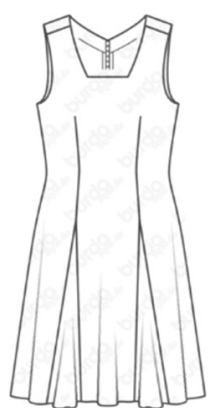

Design of a godet dress [9]

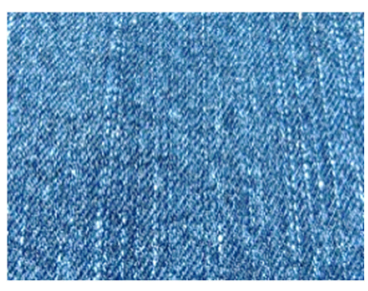

\begin{tabular}{|l|l|}
\hline Textile fabric: & Woven (Denim) \\
\hline Structure: & Twill \\
\hline Fiber: & $100 \%$ Cotton \\
\hline Weight per unit area: & $407 \mathrm{~g} / \mathrm{m}^{2}$ \\
\hline Material thickness: & $1.03 \mathrm{~mm}$ \\
\hline
\end{tabular}

B1 (right side)

Fig. 7. Design and basic data of material B1 for a Godet dress 


\subsubsection{Bending stiffness}

The bending stiffness was determined by the cantilever method using the ACPM 200 automatic bending stiffness testing device [10]. Following DIN 53362 [11], the width-related bending stiffness, see Table 1, was calculated in three directions according to equation 1.

$$
\begin{aligned}
& B=\frac{\left(g_{n} * \frac{m}{l}\right)}{b} *\left(\frac{l_{\mathrm{u}}}{2}\right)^{3} \\
& g_{\mathrm{n}} \text { Gravitational acceleration [m/ } \mathrm{s}^{2} \text { ] } \\
& \mathrm{m} \text { Mass of sample [g] } \\
& \text { I Length of sample [cm] } \\
& \text { b Width of sample [cm] } \\
& \mathrm{I}_{u} \quad \text { Length of the overhang (at } 41.5^{\circ} \text { inclination } \\
& \text { to the horizontal plane) [cm] }
\end{aligned}
$$

Table 1. Overview of the width-related bending stiffness $B$ of fabric B1

\begin{tabular}{|l|c|c|c|}
\hline \multicolumn{1}{|c|}{ Fabric B1 } & Warp & Weft & $45^{\circ}$ \\
\hline Overhang length (lü) [mm] & 60.46 & 41.91 & 60.48 \\
\hline Bending stiffness $(B)\left[\mathrm{mN}^{*} \mathrm{~cm}\right]$ & 11.40 & 3.95 & 11.34 \\
\hline
\end{tabular}

\subsubsection{Tensile behaviour}

The tensile testing machine Z2.5 "Zwicki Junior" from ZwickRoell was used for the determination of tensile stress of B1 based on DIN EN ISO 13934-1. The test sample was loaded to an upper force limit of $25 \mathrm{~N}\left(F_{\max }\right)$ at a speed of $100 \mathrm{~mm} / \mathrm{min}$. The stress-strain diagram in Figure 8 shows the different material behaviour in the three directions.

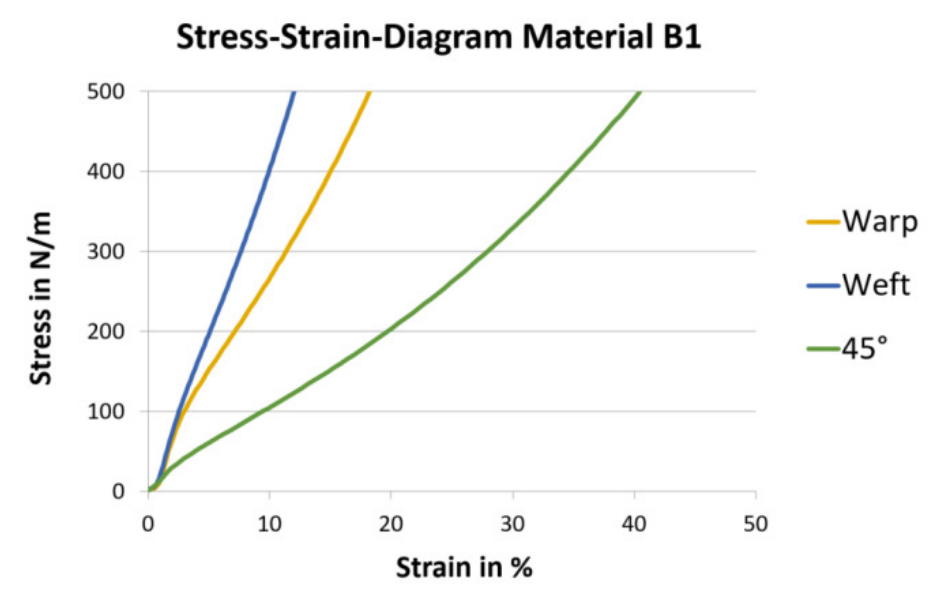

Fig. 8. Overview of the Stress-Strain Diagram of B1 in different directions

Each software for fit simulation requires specific values, which can be derived from these results.

\subsubsection{Drape test}

The drape test is an important method for the evaluation of the drape behaviour of textiles. The test device is shown in Figure 9a. A camera is mounted exactly over the center point of the carrier plate. According to DIN EN ISO $9073-9$, the circular sample $(\varnothing=300 \mathrm{~mm})$ is placed horizontally on the base plate $(\varnothing=180 \mathrm{~mm})$. Figure $9 \mathrm{~b}$ shows the recorded drape contour image, which was used to calculate the drape coefficient ( $\left.D_{\kappa}\right)$ according to equation 2. The drape coefficient of material B1 was 0.9. 

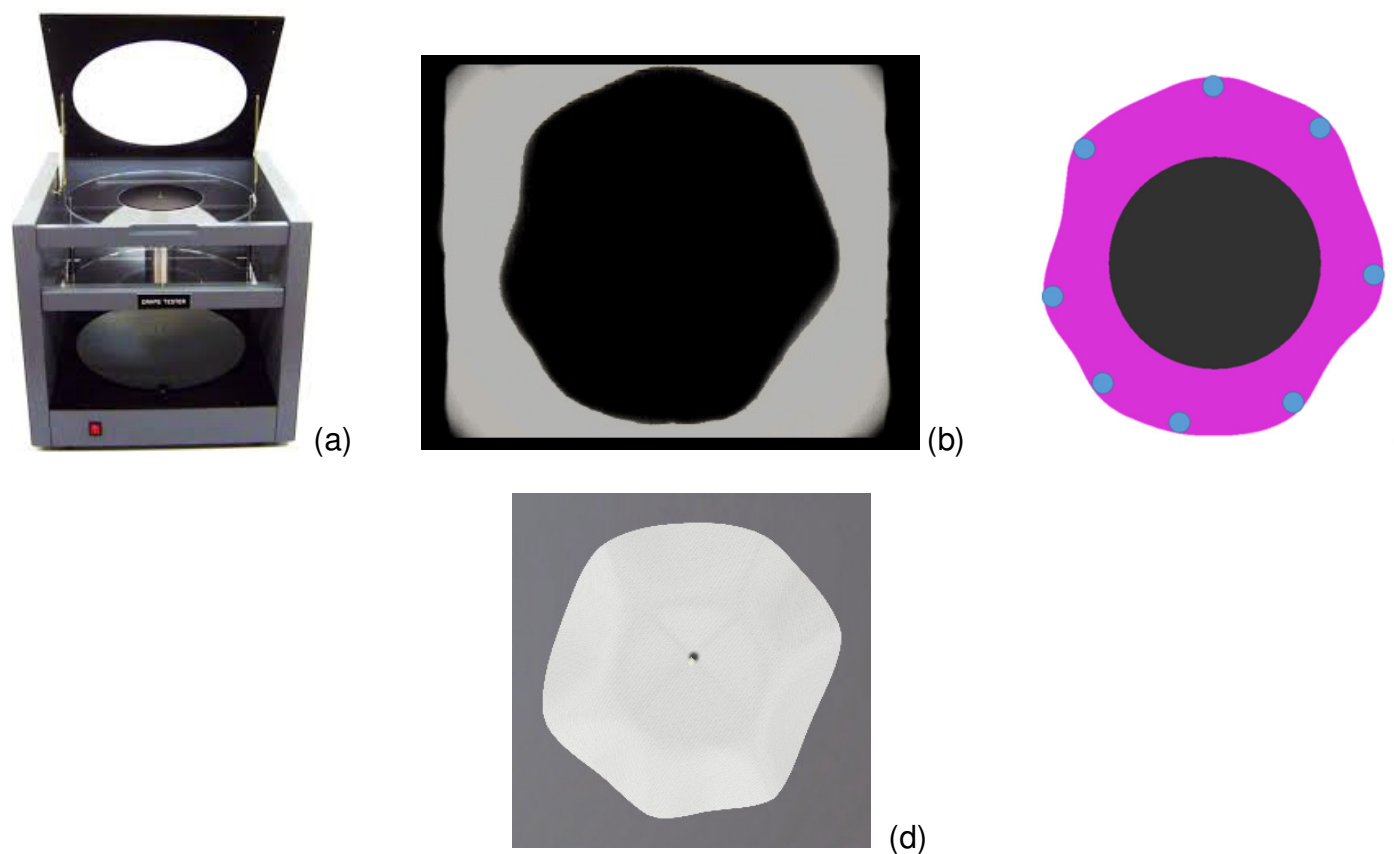

(c)

(d)

Fig. 9. Drape device and contour image of material B1, (a) drape device, (b) drape image, (c) drape contour with nodes, (d) simulated drape contour

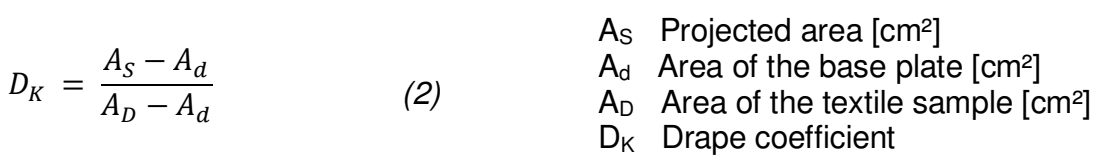

A second parameter of the drape behaviour is the number of nodes, representing wave peaks as shown in Figure 9c. When repeating the drape test, it is possible that the number of nodes can vary, but the drape coefficient must be reproducible. "Number of nodes" was further used for the comparison with the result of the dress fit control.

The material characteristics required for the fit simulation were finally prepared and integrated into the material database of the Vidya software. Vidya allows simulating the drape test for a selected material and the corresponding textile physical properties in order to compare the simulation result with the experiment. Figure 9d shows the result of the drape simulation of material B1 in Vidya with a good agreement to the experiment (Fig. 9b-c).

\subsection{D pattern development}

Based on the individual body measurements determined (see point 3.3), a Godet dress was constructed for the test person. The implementation of the basic design and the subsequent modelling was carried out in the 2D CAD software Grafis, which is frequently used in the industry. Grafis considers the individual body measurements during pattern development. Figure 10 shows the 2D pattern cuts as well as the final product made of material B1.
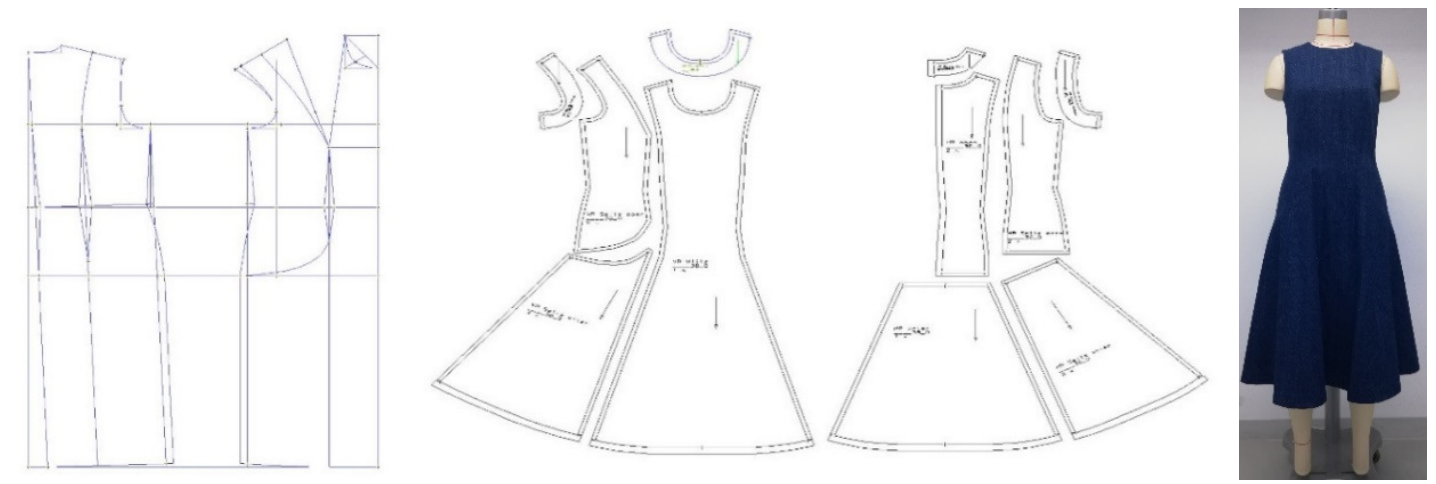

Fig. 10. 2D pattern development for dress using 2D CAD solution Grafis and the final product 


\subsection{Virtual fit control of clothing in standard pose}

The test person was scanned by using Move4D in standard pose, both with and without dress as shown in Figure 11. Move 4D is a modular photogrammetry-based 3D/4D acquisition and analysis system. It consists of a series of synchronized scanning modules that enable automatic markerless 3D acquisition of body shapes in standing and moving positions. The automatic processing software is based on data-driven body shape models and creates an artifact-free, watertight mesh reconstruction. Furthermore, there is the option to integrate a skeleton.

The data without dress were further used for automatic calculation of the body measurements according to ISO 20685-1. These were important for the individual pattern design as previously described.

Automatic processing is not useful for loose clothing like the scanned dress, because the raw data contains too many artifacts and overlaps. Therefore, the raw data was exported and processed accordingly using software for reverse engineering. With the software Geomagic, the data of the test person in the dress were prepared for further investigations. First, the lower contour of the scanned dress was compared with the contour determined in the drape test. As seen in Figure 11b, the same number of nodes (8) was observed between the scanned dress and the drape test of material (Fig. 9c). In addition,the scan data can be compared with the result of the virtual fit simulation in Vidya (see Fig. 12b).

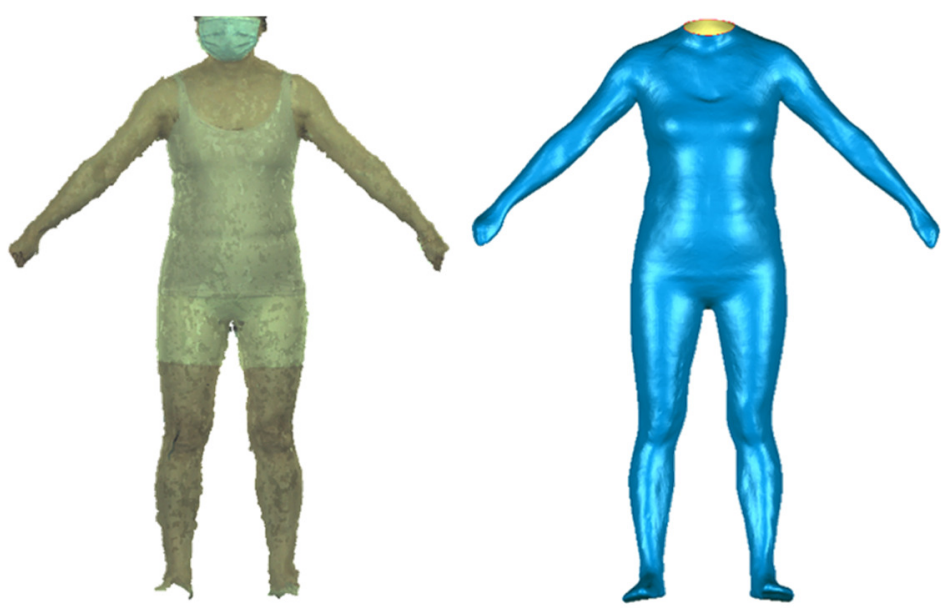

(a) Scan data without dress and automatically preparation to a watertight mesh
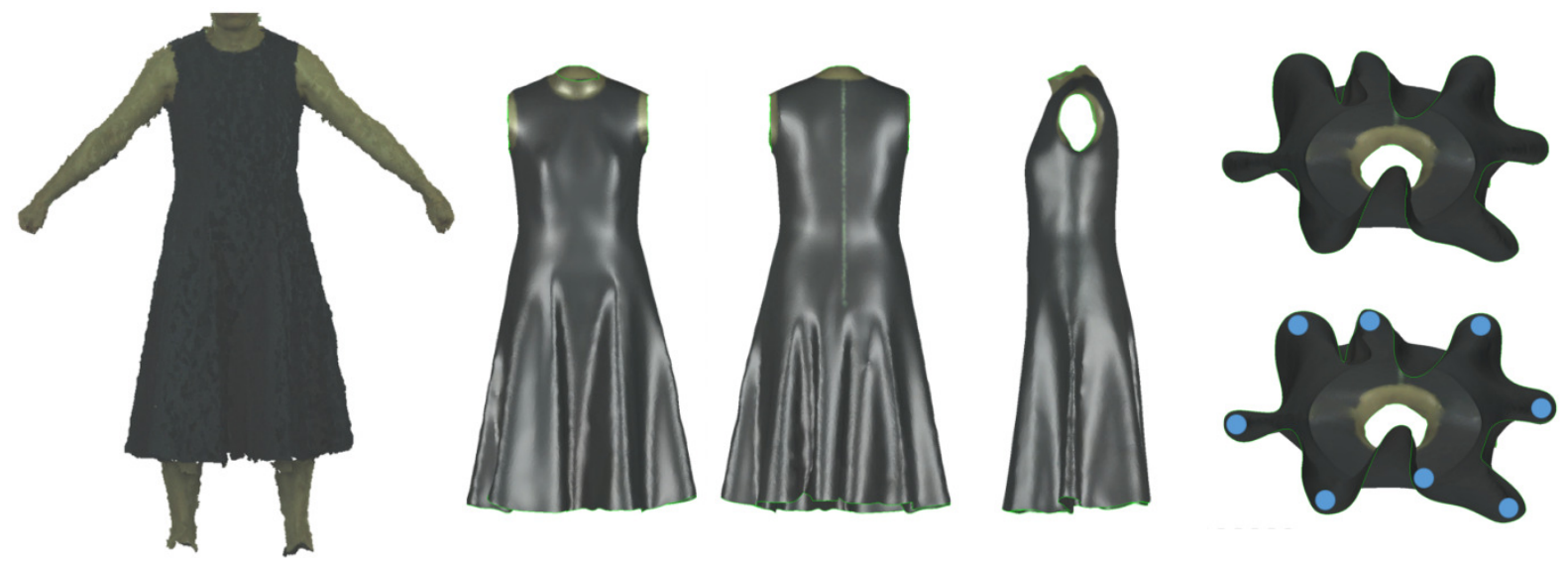

(b) Scan data with dress and preparation the data in Geomagic

Fig. 11. Scanned data of test person without (a) and with (b) dress in standard pose with additional comparison of the number of nodes in a horizontal plane at the bottom of the dress

The fit control of the dress was performed Vidya (Assyst), which allows digital 3D visualisation of garments on the individual body. The previously scanned data set of the test person in standard pose was imported into Vidya for 3D fit simulation. In addition, the developed 2D pattern cuts were implemented as an AAMA/DXF file. After assigning the sewing information and the fabric properties, the pattern cuts were simulated on the virtual test person (Fig. 12a). The shape and the number of nodes of the dress depend on the material behaviour and also on the dress length. 

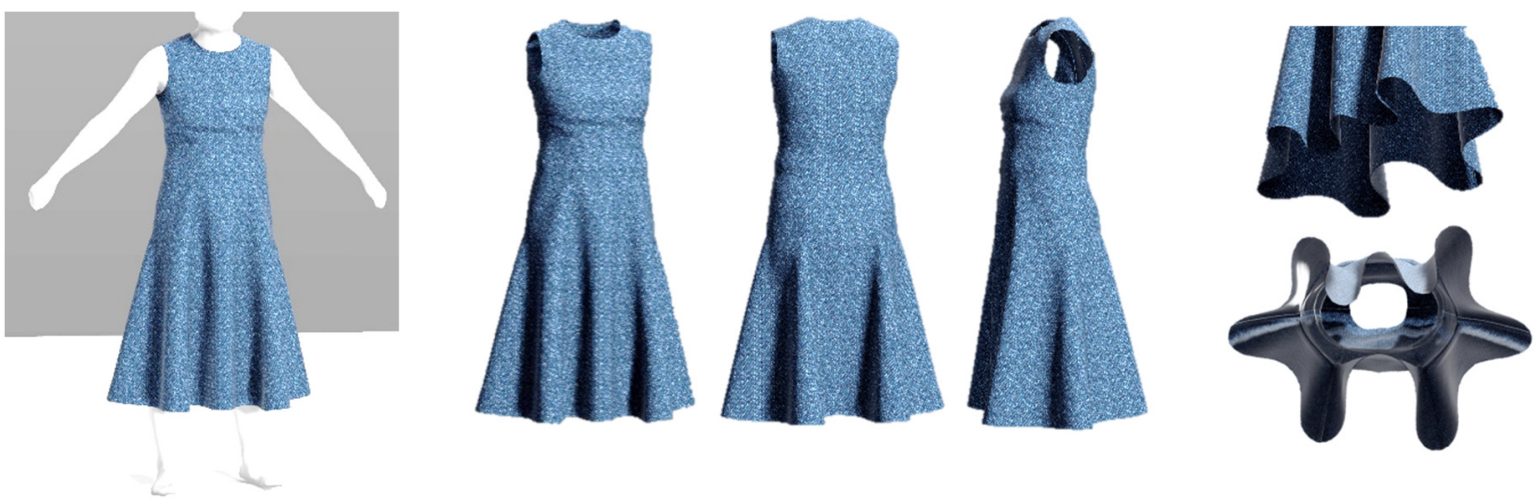

(a)
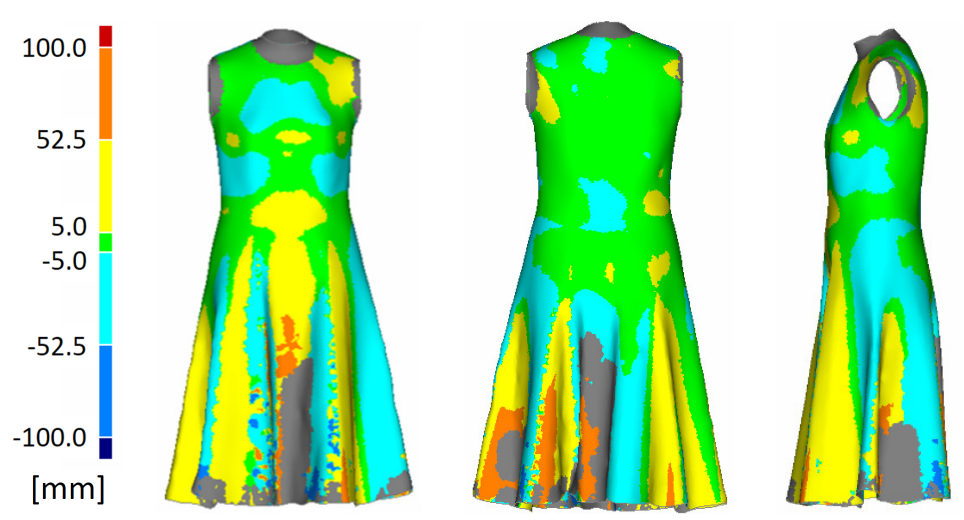

(b)

Fig. 12. (a) 3D fit simulation of dress on test person in Vidya (b) 3D analysis between fit simulation and 3D scan

Finally, the quality of the virtual fit control was checked by using software Geomagic Qualify. This program offers the possibility to compare and analyse test data with a reference model. The scanned model with dress (reference model) was compared with the model of fit simulation (test model). The test and reference model were aligned to each other in the best possible way. Figure $12 b$ shows the resulting deviations in $\mathrm{mm}$. It can be clearly seen that the large deviations were caused by a partially different draping. This was also shown by the cut surfaces (cross section) of the draped dress in the Vidya fit simulation (Fig. 12a) and the scan (Fig. 11b). The result is not unusual. This means that further analysis tools have to be developed to evaluate the simulation results.

\subsection{Virtual fit control of clothing in motion}

In a further step, the fit simulation of the clothing in motion was examined. The aim of the investigation is to compare the resulting folds in motion both from simulation and from scanning.

For this purpose, the scan data of a person in standard pose was coupled with a 23-joint skeleton and an individual kinematic human model was generated (Fig. 13a). The motion sequence or a specific walking position for the kinematic human model was taken from the scan data from Move $4 D$ (one frame). Figure $13 \mathrm{~b}$ shows a scan with the Godet dress in the examined motion (one frame). A coupling of the scan and the skeleton is problematic. The 23-joint skeleton must correspond to the surface of the scan image. Not all marker points on the body surface can be detected through the clothing (Fig. 13b). Therefore, the data of the Godet dress and the kinematic human model in the examined walking position (one frame) were imported into a coordinate system in Geomagic Qualify. The analysed penetrations between clothing and body (Fig. 13c) indicate insufficient adaptation of the body pose to the examined walking position (one frame).

The reproducibility of the movement sequences in the scan recordings can be achieved through markings on the floor for the steps or with mechanically animated dummies. 


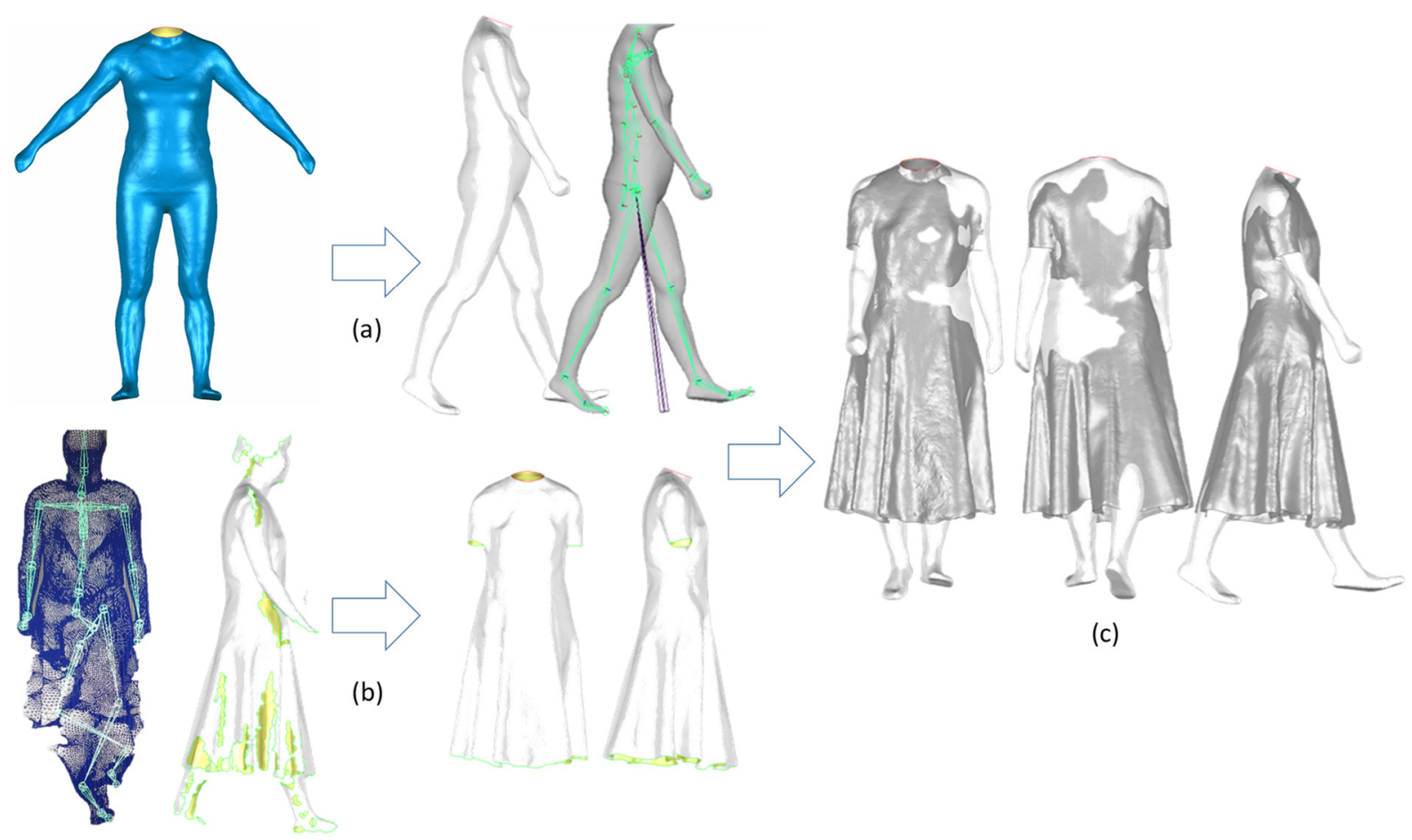

Fig. 13. Virtual fit control of the person with dress in walking: (a) Generation of an individual kinematic human model based on A-Pose (b) Incorrect integrated skeleton (scan with clothes) (c) Analysis of penetrations between clothing and body

\section{Conclusion}

In apparel industry the product development is costly. Extensive collections must be realised in evershorter time. Virtual solutions across the entire process chain from design up to prototypes are not yet "state of the art". The fit simulation is an essential part within this process chain and allows the virtual visualisation and development of garments. In addition, the virtual product should be as similar as possible to the real garment. Hence, the appropriate characterization of textile materials and derivation of a material database to achieve realistic simulations is a major focus.

Advantages of the digital linked process chain based on advanced scan solutions are as follows:

- The apparel development process is shortened through simulation solutions. Material properties are taken into account at an early stage.

- Product development in 3D on previously generated body models (scanning) enables the creation of functional clothing in specific body poses.

\section{Acknowledgment}

The research project (ZIM ZF 4008336CJ9) of the Research Community Forschungskuratorium Textil e. V. is funded by the AiF within the program "Zentrales Innovationsprogramm Mittelstand (ZIM)" of the German Federal Ministry of Economic Affairs and Energy (BMWi) by a resolution of the German Bundestag.

\section{References}

[1] Move4D, Scanning System at the Instituto de Biomecánica (IBV), www.ibv.org, accessed 2021.

[2] A. Fairfull, S. Baker, S. Warde, P. Cherns: Material intelligence for CAD, PLM and Industry 4.0, Ansys white paper, www.ansys.com, accessed 2021.

[3] E. Wendt, S. Krzywinski: Entwicklung einer Methodik zur anwendungsorientierten aerodynamischen Konstruktion textiler Flächengebilde und Funktionskleidung für SpeedSportarten (Beispiel Radrennkleidung für den Hochleistungssport) (ZIM ZF 4008336CJ9). Dresden: Technische Universität Dresden, Institut für Textil- und Bekleidungstechnik, Abschlussbericht, 2021. 
[4] J. Siegmund, S. Krzywinski: Seriennahe Entwicklung und prototypische Fertigung marktfähiger aerodynamisch optimierter Sportanzüge aus funktionellen Textilien zur Anwendung in Vertikalwindtunneln (ZIM ZF 4008314CJ7). Dresden: Technische Universität Dresden, Institut für Textil- und Bekleidungstechnik, Abschlussbericht, 2021.

[5] SMPL, A Skinned Mutli-Person Linear Model, smpl.is.tue.mpg.de, accessed 2021.

[6] S. Krzywinski: Verbindung von Design und Konstruktion in der textilen Konfektion unter Anwendung von CAE. Dresden, Technische Universität Dresden, Fakultät Maschinenwesen, Habilitation, 2005.

[7] J. Siegmund: Erarbeitung virtueller Menschmodelle als Konstruktionswerkzeug zur 3DProduktentwicklung in der Bekleidungsindustrie. Dresden, Technische Universität Dresden, Fakultät Maschinenwesen, Dissertation, 20013.

[8] P. Volino, N. Magnenat-Thalmann: Virtual clothing: theory and practice. Springer-Verlag Berlin Heidelberg, 2000, doi: 10.1007/978-3-642-57278-4.

[9] Design of a Godet Dress, www.burda.style, accessed 2021.

[10] M. Seif: Bereitstellung von Materialkennwerten für die Simulation von Bekleidungsprodukten. Dresden, Technische Universität Dresden, Fakultät Maschinenwesen, Dissertation, 2007.

[11] Norm DIN 53362:2003-10. Prüfung von Kunststoff-Folien und von textilen Flächengebilden (außer Vliesstoffe), mit oder ohne Deckschicht aus Kunststoff - Bestimmung der Biegesteifigkeit Verfahren nach Cantilever. 